\title{
Higher Molecular Weight Polyethylene Glycol Increases Cell Proliferation While Improving Barrier Function in an In Vitro Colon Cancer Model
}

\author{
Shruthi Bharadwaj, ${ }^{1,2}$ Ramana Vishnubhotla, ${ }^{3}$ Sun Shan, ${ }^{3}$ Chinmay Chauhan, ${ }^{3}$ \\ Michael Cho, ${ }^{3}$ and Sarah C. Glover ${ }^{1,2}$ \\ ${ }^{1}$ Department of Medicine, University of Florida, 1600 SW Archer Road, Box 100214, Gainesville, FL 32608, USA \\ ${ }^{2}$ Department of Biomedical Engineering, Gainesville, FL 32610, University of Florida, USA \\ ${ }^{3}$ Department of Bioengineering, University of Illinois at Chicago, Chicago, IL 60607, USA
}

Correspondence should be addressed to Sarah C. Glover, sarah.glover@medicine.ufl.edu

Received 1 July 2011; Accepted 4 August 2011

Academic Editor: Rumiana Koynova

Copyright (C) 2011 Shruthi Bharadwaj et al. This is an open access article distributed under the Creative Commons Attribution License, which permits unrestricted use, distribution, and reproduction in any medium, provided the original work is properly cited.

Polyethylene glycol (PEG) has been previously shown to protect against enteric pathogens and prevent colon cancer invasion. To determine if PEG could indeed protect against previously observed pro-invasive effects of commensal E. coli and EPEC, Caco-2 cells grown in an in vitro model of colon cancer were infected with strains of human commensal E. coli or EPEC and treated with 10\% PEG 3350, PEG 8000, and PEG 20,000, respectively. At 24 hours after infection, MMP-1 and MMP-13 activities, cell cluster thickness, depth of invasion, and proliferation were determined using standard molecular biology techniques and advanced imaging. We found that higher molecular weight PEG, especially PEG 8000 and 20,000, regardless of bacterial infection, increased proliferation and depth of invasion although a decrease in cellular density and MMP-1 activity was also noted. Maximum proliferation and depth of invasion of Caco-2 cells was observed in scaffolds treated with a combination of commensal E. coli strain, HS4 and PEG 8000. In conclusion, we found that PEG 8000 increased cell proliferation and led to the preservation of cell density in cells treated with commensal bacteria. This is important, because the preservation of a proliferative response in colon cancer results in a more chemo-responsive tumor.

\section{Introduction}

Colon cancer remains the third leading cause of cancer mortality worldwide [1]. Colon cancers are unique in so far as they arise in a microenvironment teaming with bacteria. In the absence of colorectal cancer or colonic inflammation, intestinal bacteria are involved in the promotion of nutrition, lymphoid development, competition with pathogenic bacteria, and with tissue repair [2-5]. They may also play a role in oncologic surveillance and induce apoptosis to prevent abnormal colonic proliferation [6].

The symbiotic relationship between the cells of the colon and the commensal bacteria adjacent to them is normally maintained by five major "firewalls." These are the mucus layer atop of the epithelial cells $[7,8]$, the epithelia themselves, subepithelial macrophages, dendritic cells, and mesenteric lymph nodes [9]. However, with the occurrence of colon cancer, barrier function between the epithelial cells and commensal bacteria surrounding them is lost. As such, these previously noninvasive bacteria gain the ability to invade into the epithelial layer both enzymatically and nonenzymatically, further disrupting cellular function.

Polyethylene glycol (PEG) is used clinically to treat chronic constipation and to prep individuals for colonoscopy and various other gut imaging procedures [10, 11]. PEG has been used as a vehicle to successfully deliver chemotherapy in an in vitro murine model of colon cancer [12]. Recently, it has been noted that PEG has profound chemopreventive properties in both experimental models of colon carcinogenesis [13] and in human population studies [14]. PEG's ability to act as a chemo-preventive agent is most likely due to its ability to induce apoptosis in damaged cells $[13,15,16]$. 
Furthermore, PEG has also been shown to reinforce epithelial barrier function in the colon in experimental models of colitis [17]. PEG molecules have been known to adhere to proteins and surfactant phospholipids thereby forming barrier-like structures [18-20].

While the most widely available form of PEG is the molecular weight of 3350, both high and low molecular weight forms of PEG have been shown to be of potential clinical importance. For example, PEG 15-20, a low molecular weight PEG, has been shown to protect against radiation-induced intestinal injury via its ability to bind lipid rafts and prevent their coalescence [21]. Further, PEG 8000 has demonstrated functionality as a barrier inhibiting interactions between colonizing microbes and their epithelial cell targets, thereby forming a mucin-like layer [22].

Under specific conditions the commensal bacterial strains are able to overcome protective host responses and exert pathologic effects that may aid in the onset of colon cancer [23]. Pathogenic E. coli strains, specifically Enteropathogenic E. coli (EPEC), have been known to attach to intestinal epithelial cells and cause epithelial cytoskeletal changes and disruption of surrounding microvilli [24]. Similarly, the invasive characteristics of malignant colonic epithelia further disrupt the gut barrier function in the event of colon cancer.

On the basis of the literature to date, we hypothesized that PEG might also help restore the symbiotic relationship between commensal bacteria and malignant colonic epithelia, thereby decreasing their proinvasiveness with the occurrence of colon cancer. To test this, we utilized an in vitro colon cancer model, PEG of various molecular weights (MW 3350, 8000, and 20,000) and both commensal and pathogenic bacteria as described below.

\section{Experimental Procedures}

2.1. Reagents and Supplies. All cell culture reagents excluding fetal bovine serum (FBS), from Gemini Bio-products (West Sacramento, Calif, USA), were obtained from Mediatech, Inc. (Herndon, VA). Caco-2 cells were purchased from ATCC. Cells were maintained in dishware from BD Falcon (Lincoln Park, NJ, USA). Type I rat tail collagen was purchased from BD Bioscience (Bedford, Mass, USA). MTT assay components and phalloidin was obtained from Invitrogen (Carlsbad, Calif, USA). All other supplies were molecular biology grade and were from Fisher Scientific (Pittsburg, PA, USA).

2.2. Cell Culture. Caco-2 cells were cultured in D-MEM/F-12 50/50 1X with L-glutamine and 15 mM HEPES supplemented with $10 \%$ fetal bovine serum and were incubated at $37^{\circ} \mathrm{C}$ in a $5 \% \mathrm{CO}_{2}$ atmosphere.

2.3. Creation of $3 D$ Scaffolds. Type I collagen gel scaffolds were created and seeded with cells. Type I collagen was placed into a solution of $0.1 \mathrm{M}$ sodium hydroxide combined with 10X Hanks buffered salt solution (HBSS). Media was added to this mixture and then was neutralized using $0.1 \mathrm{M}$ acetic acid to create a gel of $1.2 \mathrm{mg} / \mathrm{mL} .800 \mu \mathrm{L}$ of the collagen gel mixture was placed into each well of a 12 -well plate and incubated for one hour prior to seeding. Cells were seeded at a density of 250.000 cells $/ \mathrm{cm}^{2}$.

2.4. Infection of Scaffolds. Scaffolds were infected with either commensal or pathogenic E. coli strains. Bacteria were grown overnight in LB Broth on a bacterial shaker at $37^{\circ} \mathrm{C}$. $1 \mathrm{~mL}$ of bacterial culture was added to $5 \mathrm{~mL}$ of serumfree media and placed on a shaker at $37^{\circ} \mathrm{C}$ for two hours. Bacterial concentration was determined by measuring the optical density (OD) at $600 \mathrm{~nm}$. Bacteria were added to the scaffolds at an MOI = 30 per cell in serum-free DMEM/F-12. Samples were then incubated at $37^{\circ} \mathrm{C}$ for two hours. Bacteria containing media was then removed and the scaffolds were treated with gentamicin at a concentration of $50 \mu \mathrm{g} / \mathrm{mL}$ for two hours. Following removal of antibiotic containing media, scaffolds were incubated overnight in fresh serumfree media containing $10 \mu \mathrm{g} / \mathrm{mL}$ gentamicin.

2.5. Multiphoton Microscopy. Multiphoton imaging holds inherent advantages for imaging living tissues by improving depth penetration and reducing photodamage [25]. Twophoton or multiphoton imaging allows the mapping of fluorophore distribution inside tissue down to a depth of over $500 \mu \mathrm{m}$ providing subcellular level tissue morphological information. Samples were seeded onto $1.2 \mathrm{mg} / \mathrm{mL}$ concentration type I collagen with an approximate scaffold thickness of $5 \mathrm{~mm}$ and imaged using multiphoton microscopy.

Cells were seeded onto scaffolds and infected according to the protocol mentioned before. Scaffolds were then washed with phosphate buffered saline (PBS), fixed overnight with $10 \%$ neutral buffered formalin. Scaffolds were then blocked for 30 minutes with serum free protein block and stained for actin using phalloidin. Scaffolds were then incubated with $300 \mathrm{nM}$ DAPI in PBS for 5 minutes to stain nuclei.

Seeded cells and actin cytoskeleton were imaged by a laser scanning multiphoton confocal microscope with 60x oil objective (NA = 1.40). DAPI was visualized by using multiphoton laser excitation at $700 \mathrm{~nm}$ and emission at $450 \mathrm{~nm}$, for which the femtosecond laser beam $(80 \mathrm{MHz}, 0.5 \mathrm{~mW})$ pumped from a mode-locked titanium sapphire laser (MaiTai, Spectra-Physics Inc., Calif, USA), was coupled with visible laser (Bio-Rad, UK) into an inverted laser scanning confocal microscope (Nikon TE200-U, Japan). Reflection signals from the collagen fibers were excited and acquired at a wavelength of $488 \mathrm{~nm}$.

2.6. Multiphoton Image Analysis. Multiphoton images were analyzed to determine depth of invasion, cluster thickness, and cluster density.

To determine depth of invasion, commercially available software, Lasersharp 2000, was used. Briefly, the lowest point with actin (red) and DAPI (blue) staining within the scaffold was measured for each sample.

To determine the cluster thickness, the multiphoton images were tilted to visualize the scaffold in $z$-direction. The thickness was determined by measuring a portion of the scaffold where the cells were most densely packed. 
To measure the cluster density, the number of cells were counted in a $100 \mu \mathrm{m} \times 100 \mu \mathrm{m}$ area within the scaffold.

2.7. Proliferation Assay. Cell proliferation was determined using a standard MTT assay kit. To determine the degree of proliferation following treatment with commensal E. coli strains, EPEC, or following treatment with each of the various bacteria in the presence of PEG 3350, PEG 8000, or PEG 20,000, we used a standard MTT assay. This particular method is useful for determining proliferation in these types of $3 \mathrm{D}$ cultures, because it does not require disruption of the cells from their scaffold. Each experiment was performed a minimum of three times.

All components used were free of phenol red, since it may interfere with the accuracy of the assay. To do this, the media was removed from each chamber and the gels were washed with PBS. $100 \mu \mathrm{L}$ fresh media and $20 \mu \mathrm{L}$ of dye ((3-(4,5-dimethylthiazol-2-yl)-2,5-diphenyltetrazolium bromide) in PBS was added to each chamber. The gels were incubated at $37^{\circ} \mathrm{C}$ for four hours. After four hours, $50 \mu \mathrm{L}$ from each chamber was added to $100 \mu \mathrm{L}$ of dimethyl sulfoxide (DMSO) in a small vial and incubated for fifteen minutes. Absorbance readings were taken at $550 \mathrm{~nm}$ for each sample to determine the cell proliferation values.

2.8. Matrix Metalloprotease Activity. MMP-1 and 13 activities were determined using a commercially available fluorometric assay designed to quantitatively measure enzyme activity. Briefly a monoclonal antibody specific for each MMP1 or MMP-13 was precoated onto a microplate. Standards and samples were placed into the wells, and the MMP of interest was bound by the immobilized antibody. After washing away unbound substances, an activation reagent (APMA) was added to standards and samples. Following a wash, a fluorogenic substrate linked to a quencher molecule is added. Active enzymes cleave the peptide linker between the fluorophore and the quencher molecule. A fluorescent signal proportional to the amount of enzyme activity is produced due to this cleavage.

\section{Results}

3.1. Higher MW PEGs Increase Proliferation. Cellular proliferation increased slightly over control in response to the two commensal E. coli strains, 264 and HS4 (Figure 1). Specifically, proliferation increased 1.2 -fold in response to treatment with strain 261 and 1.5-fold in response to treatment with HS4. HS4 bacterial strain by itself increased proliferation. However, proliferation was attenuated by incubation of the cells with EPEC that led to a reduction in cellular proliferation as compared with control by nearly two thirds.

PEG 8000 and PEG 20,000 alone increased cell proliferation four and three fold over control, respectively. Treatment with PEG 3350 did not significantly alter proliferation.

When cells were treated with bacteria and PEG, cellular proliferation increased significantly. Specifically, combination of PEG 8000 and HS4 increased proliferation by sixfold and combination of HS4 with PEG 20,000 increased proliferation twofold. Proliferation remained constant when

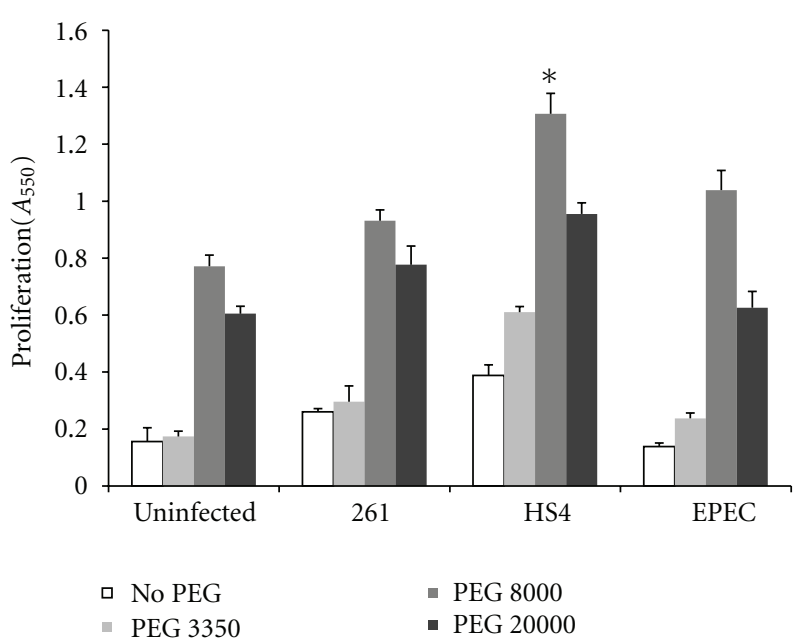

FIGURE 1: PEG 8000 increases proliferation of Caco-2. Proliferation of cells on the scaffold was observed using MTT proliferation assay as described in Methods. Higher molecular weight PEGs, 8000 and 20,000, increased proliferation regardless of bacterial infection. Maximum proliferation was noted when cells were treated with HS4 and PEG 8000. Standard Student's $t$-test was applied to the samples and $P<0.05$ were obtained for samples treated with PEG 8000 and HS4 indicated by an asterisk $(*)$.

treated with PEG 3350 regardless of bacterial strain (Figure 1).

3.2. Higher MW PEGs Decrease Cell Cluster Thickness. As described in the methods section of this paper, cell cluster thickness refers to the bulk of cells in a scaffold. In collagen scaffolds infected with both bacterial strains and PEG 8000 cells were more dispersed and were not as tightly packed as seen in the control scaffolds treated with PEG 8000 alone). This was particularly true when samples were infected with HS4 (Figure 2). Collagen scaffolds were divided into approximately 16 stacks (each stack was approximately $5 \mu \mathrm{m}$ ) to better visualize the penetration depth. In the control sample, which was treated with PEG 8000 alone, cells penetrated to approximately 10 stacks $(\sim 50 \mu \mathrm{m})$. When incubated with bacterial strain 261 , decreased cluster thickness to approximately 8 stacks $(40 \mu \mathrm{m})$. When infected with bacterial stain HS4, cluster thickness remained fairly constant in spite of higher proliferation rate (Figure 1).

Cell cluster thickness decreased when treated with different molecular weight PEG (Figure 3). The control sample where no PEG was added, the cluster thickness was approximately $100 \mu \mathrm{m}$. When samples were treated with PEG 3350, the cluster thickness decreased to approximately $70 \mu \mathrm{m}$, and when treated with PEG 8000, the cluster thickness decreased to approximately $50 \mu \mathrm{m}$.

3.3. Quantitative Analysis of Depth of Invasion. As described in the methods section of this paper, depth of invasion refers to how far the cells penetrate into the scaffold. The difference between this and cell cluster thickness is that in depth of invasion describes the lowest point in the scaffold at which the cellular signal is detected. The quantification of depth 


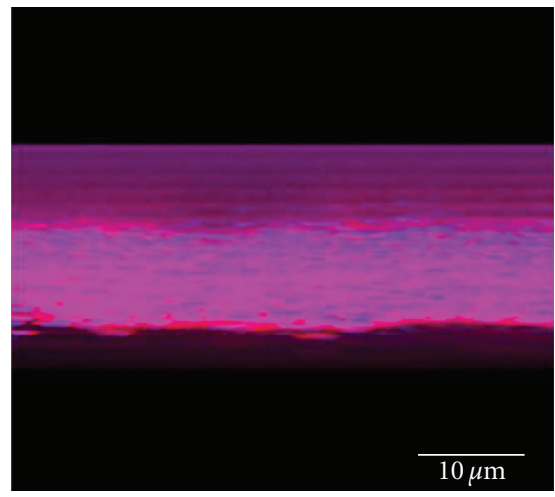

(a)

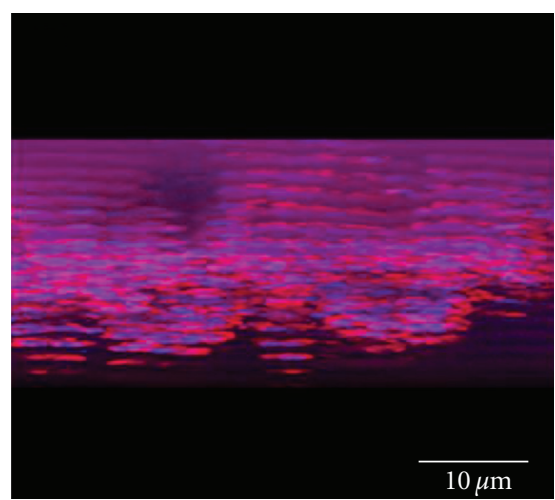

(c)

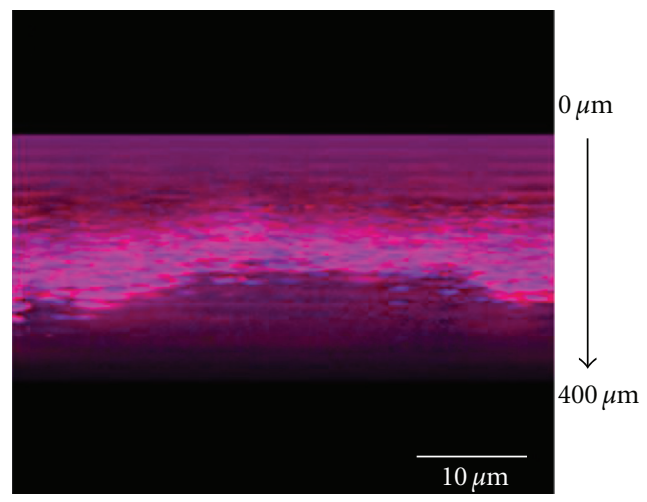

(b)

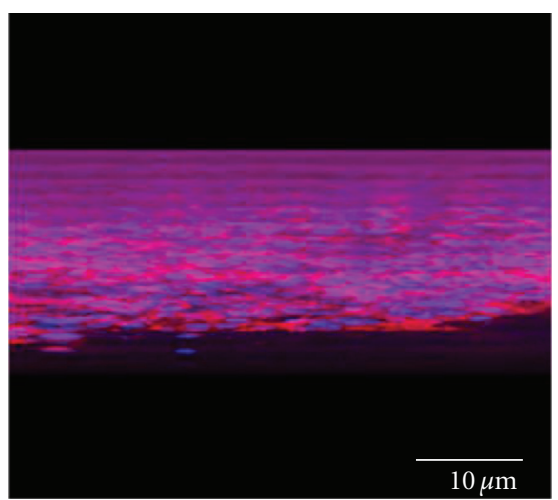

(d)

FIGURE 2: Multiphoton images of Caco-2 cells treated with PEG 8000 and various bacterial strains. Uninfected cells were served as control (a). Caco-2 cells were infected with commensal strains, 261 and HS4 (b, c), and EPEC (d). The maximum depth of invasion was seen in scaffolds infected with HS4. The overall thickness of the scaffold was approximately $400 \mu \mathrm{m}$. The actin filaments are shown in red and the DAPI staining of nuclei are shown in blue. Bar length $=10 \mu \mathrm{m}$.

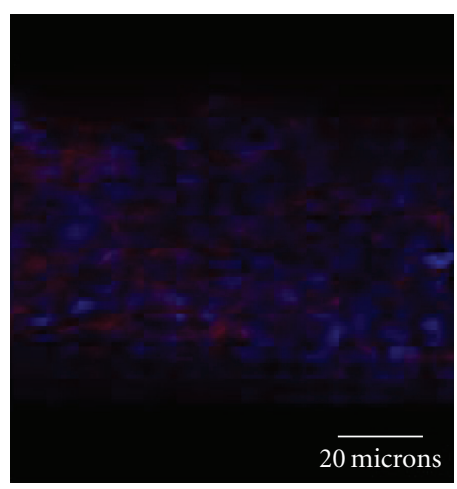

(a)

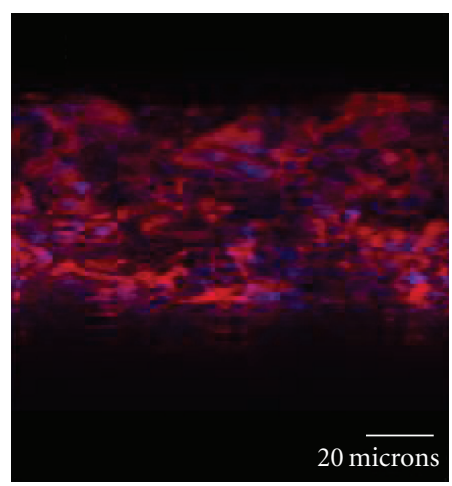

(b)

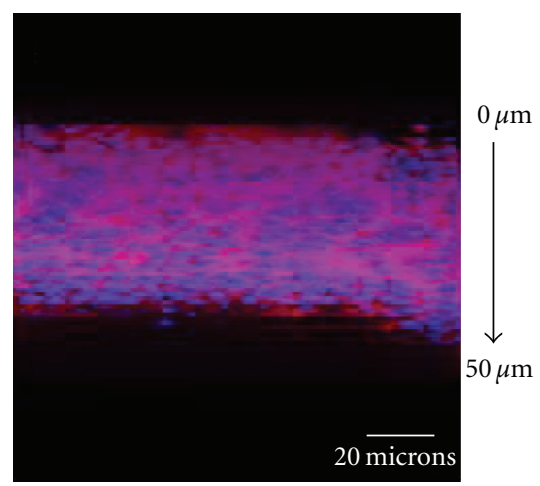

(c)

FIGURE 3: Multi-photon Images of Caco-2 cells treated with various molecular weight PEGs (a) Untreated cells were served as control. (b, c) Caco-2 cells were treated with PEG 3350 and PEG 8000 respectively. Cluster thickness decreased when treated with higher molecular weight PEG. The cluster thickness when treated with PEG 8000 was approximately $50 \mu \mathrm{m}$. The actin filaments are shown in red and DAPI staining of nuclei is in blue. Bar length $=20 \mu \mathrm{m}$.

of invasion is essential in determining the proinvasive effect of each of the variables on Caco- 2 cell behavior. In the data presented above, it appears that Caco-2 cells may be more aggressive when treated with higher molecular weight PEG and the commensal bacterial strain, HS4 (Figures 1 and 2).
A threefold increase in cell penetration depth was observed in samples infected with either commensal or pathogenic bacteria alone. Also, higher molecular weight PEG alone, especially PEG 8000, increased depth of invasion by twofold (Figure 4). 


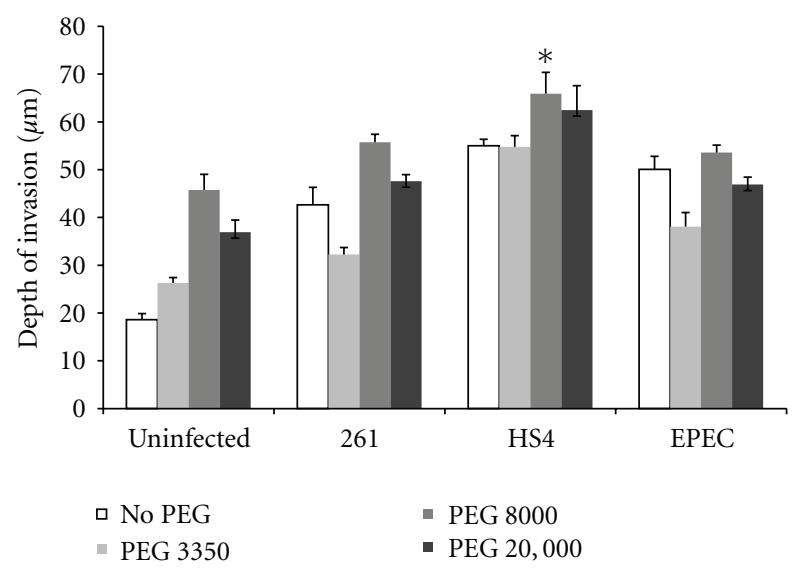

Figure 4: Quantitative analysis of depth of invasion. Depth of invasion was analyzed as described in Methods. All three PEGs and all three bacterial strains independently increased depth of invasion. A decrease in invasion was noted when samples were treated with PEG 3350 and either 261 or EPEC. Treatment with any bacterial strain and either PEG 8000 or PEG 20,000 led to increased invasion. The greatest increase was seen when samples were treated with PEG 8000 and HS4. Standard Student's $t$-test was applied to the samples and the $P<0.05$ were obtained for samples treated with PEG 8000 and HS4 indicated by an asterisk $(*)$.

Samples exposed to both PEG and bacterial condition slightly increased the invasion depth of Caco-2 cells compared to samples exposed to PEG or bacteria alone. Maximum invasion of $65 \mu \mathrm{m}$ was observed in samples treated with PEG 8000 and infected with HS4.

3.4. Quantitative Analysis of Cell Density. As described in the methods section of this paper, cell density refers to the number of cells within a given area. The difference between this and cell cluster thickness is that cell density is the number of cells within a $100 \mathrm{~mm} \times 100 \mathrm{~mm}$ (both in $Z$ and $Y$ directions) scaffold matrix whereas cell cluster thickness was measured in $Z$-direction only.

Each bacterial strain and each PEG, when observed independently, significantly reduced the number of cells in a given area (Figure 5). This was particularly true when cellcontaining scaffolds were infected with the commensal $E$. coli, HS4. Specifically, a sixfold reduction in cell density was observed. Cell density in samples infected with either 261 or EPEC was reduced by $50 \%$ relative to control. Treatment with either PEG 3350 or PEG 8000 led to similar reduction in cell density, whereas treatment with PEG 20,000 decreased the cell density by approximately $50 \%$.

The combination of PEG with bacterial strains increased cell density. This was particularly true when cells were treated with a combination of PEG 8000 and HS4, which led to a threefold increase in cell density when compared to samples infected with HS4 alone (Figure 5).

3.5. PEG 8000 Decreases MMP-1 Activity in Cells Expose to Commensal and Pathogenic E. coli. Since MMP-1 is involved in the initial breakdown of collagen during tumor growth

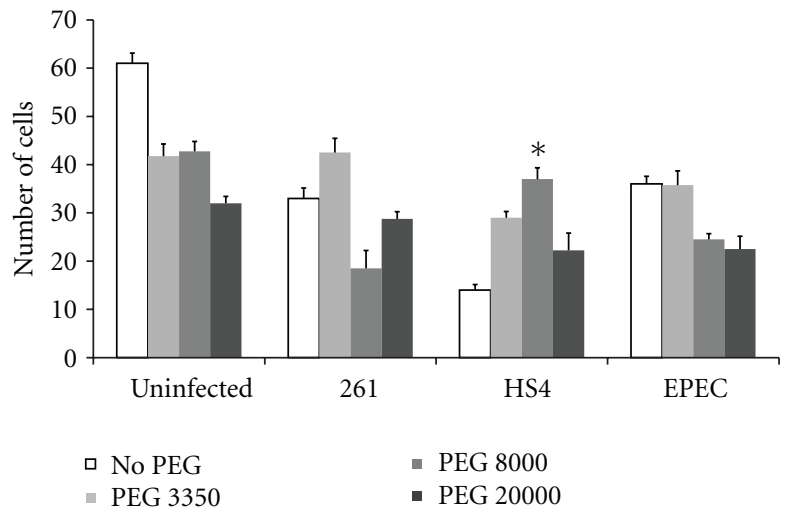

Figure 5: Quantitative analysis of cluster density. Cell density analysis was performed as described in Methods. Cell density decreased in response to independent treatment with any of the three PEGs or bacterial strains. When treated with PEG 3350 and the two commensal bacterial strains, there was an increase in cell density. Cell density recovery was most persevered when treated with PEG 8000 and HS4. PEG 20,000 did not preserve cell density when cells were treated with any of the three bacterial strains. Standard Student's $t$-test was applied to the samples and the $P<0.05$ were obtained for samples treated with PEG 8000 and HS4 indicated by an asterisk $(*)$.

and invasion [26], an increase in collagenase activity is expected when cells become more invasive. In our model system, PEG 3350 and PEG 20,000 increased MMP-1 activity in uninfected samples. However, a decrease in MMP-1 activity was observed when samples were treated with PEG 8000 alone. Infecting with bacterial strains alone did not significantly reduce MMP-1 activity. However, treating samples with a combination of PEG and any of the two commensal bacterial strains, 261 and HS4, reduced MMP-1 activity significantly. Specifically, a greater than twelvefold decrease in MMP-1 activity was observed in samples infected with commensal bacterial strain (HS4 or 261) and treated with PEG 3350 or PEG 8000 (Figure 6). While PEG 3350 and PEG 20,000 did lead to a slight decrease in MMP-1 activity during EPEC infection, only PEG 8000 led to a true decrease in activity, which was nearly fivefold over control.

3.6. PEG Has No Effect on MMP-13 Activity. No significant change in MMP-13 activity was observed when infected with commensal and pathogenic E. coli strains alone. A slight increase in MMP-13 activity was observed in samples treated with PEG alone. This was especially true when samples were treated with PEG 20,000 (Figure 7). Infection with commensal bacteria or EPEC slightly decreased MMP-13 activity. This was not statistically significant.

\section{Discussion and Conclusion}

In this study, we demonstrated that cell proliferation and depth of invasion were most increased when treated with higher molecular weight PEGs and commensal bacteria. These findings were somewhat surprising to us but were 


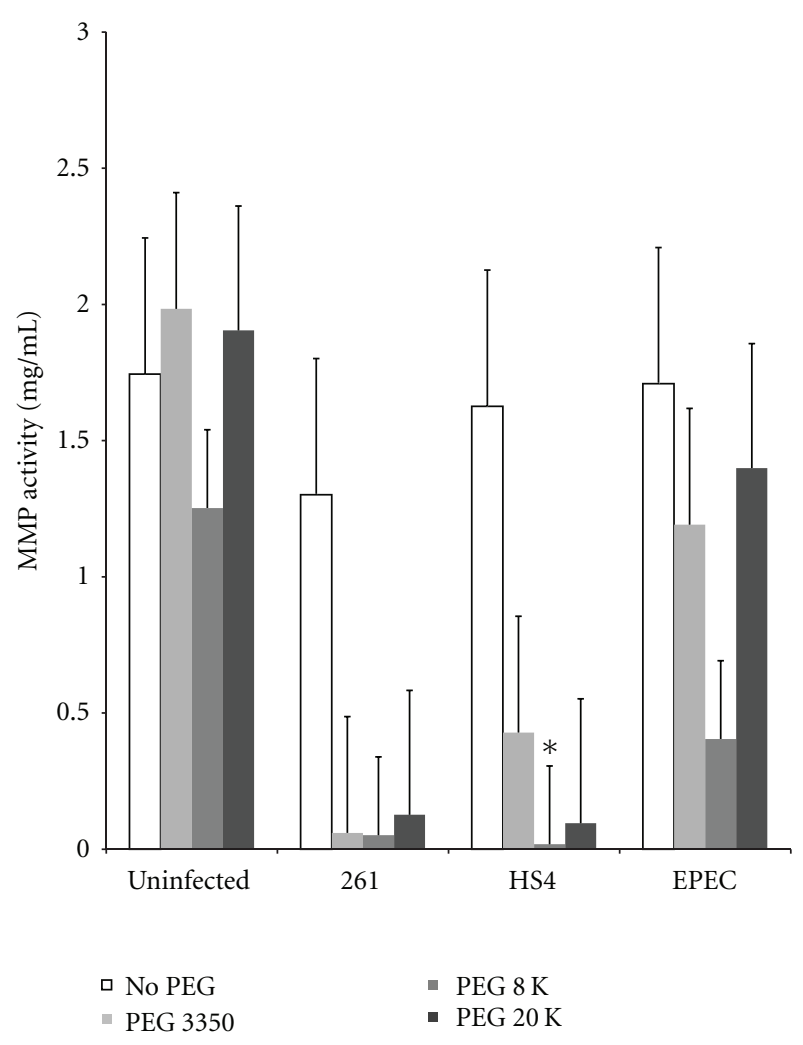

Figure 6: MMP-1 Activity of Caco-2 cells infected with commensal and pathogenic bacteria and treated with 10\% PEG. MMP-1 activity in cells treated with commensal bacterial strains were comparatively lower than when infected with pathogenic (EPEC). PEG significantly reduced the MMP-1 activity when infected with bacteria (both commensal and pathogenic). A 12-fold decrease in MMP-1 activity was noted in samples treated with PEG 8000 and infected with commensal bacteria. Standard Student's $t$-test was applied to the samples and the $P<0.05$ were obtained for samples treated with PEG 8000 and HS4 indicated by an asterisk (*).

balanced by our finding of decreased cluster thickness, that is, a decrease in the number of cells in a given area and decreased MMP-1 activity. We hypothesize that this occurred because of the structural properties of these higher molecular weight PEGs rather than because of their molecular weight per se.

The structural properties of PEG act by increasing the volume of macromolecules surrounding the cells. Increasing the amount of macromolecules surrounding the cells aids in the formation of bundled F-actin [27]. Also, in the presence of inert macromolecular "crowders" it has been shown that the association rate constant of actin monomers to an actin filament increases [28]. Furthermore, the bundling of actins has been shown to increase proliferation in colonic epithelial cells [29]. Hence, it is safe to assume that the overcrowding caused by PEG molecules aids in actin bundling which in turn increases the proliferation rate of Caco- 2 cells. The proliferative response we observed in this in vitro model in response to the two commensal strains of E. coli and to EPEC is consistent with previously published results [30]. The slight reduction in proliferation in response to EPEC is

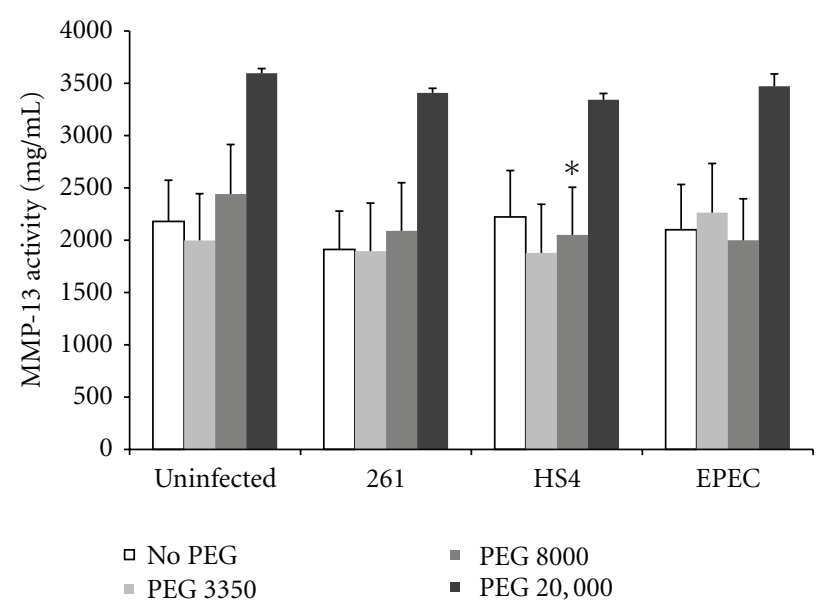

Figure 7: MMP-13 activity for Caco-2 cells infected with commensal and pathogenic bacteria and treated with 10\% PEG. There was no change in MMP-13 activity in samples treated with either PEG 3350 or PEG 8000 as compared to control regardless of infection status. Infection with commensal bacteria or EPEC slightly decreased the MMP-13 activity. This was not statistically significant. Standard Student's $t$-test was applied to the samples and the $P<0.05$ were obtained for samples treated with PEG 8000 and HS4 indicated by an asterisk $(*)$.

also not surprising considering that EPEC is known to cause cellular apoptosis [31-33].

With the increased in cell proliferation, an increase in depth of invasion of Caco-2 cells was also observed. But this is not important and is merely reflective of increased proliferation. What is more important is that cell cluster thickness decreased in response to a high molecular weight PEG milieu. This is critical, because the cells are packed more tightly. In other words, there is evidence suggesting that gut barrier function is being restored by higher molecular weight PEGS, particularly PEG 8000 . This is supported by a recent study in a rat colitis model that demonstrated that PEG increased colonic surface hydrophobicity, diminished luminal bacterial load, and reduced chemically induced mucosal damage and inflammation [17].

In summary, we have demonstrated that a significant increase in proliferative response occurs in an in vitro colon cancer model when treated with PEG 8000 and commensal E. coli strains. Our finding suggests that PEG 8000 may potentially be a valuable therapeutic agent that could be used in conjunction with standard chemotherapy. Further studies will be needed to understand the protective role of high molecular weight PEGs, particularly PEG 8000, in colon cancer and their potential for making tumors more chemosensitive by increasing cell proliferation.

\section{Funding}

The University of Illinois GILD and the National Institutes of Health (1 RO1 CA113975-A2) funded this work. 


\section{References}

[1] A. Jemal, R. Siegel, J. Xu, and E. Ward, "Cancer statistics, 2010," CA Cancer Journal for Clinicians, vol. 60, no. 5, pp. 277$300,2010$.

[2] E. Slack, S. Hapfelmeier, B. Stecher et al., "Innate and adaptive immunity cooperate flexibly to maintain host-microbiota mutualism," Science, vol. 325, no. 5940, pp. 617-620, 2009.

[3] P. L. M. J. Ogra, M. E. Lamm, W. Strober, J. Bienenstock, and J. McGhee, Eds., Mucosal Immunology, Academic Press, New York, NY, USA, 1999.

[4] H. G. Boman, "Innate immunity and the normal microflora," Immunological Reviews, vol. 173, pp. 5-16, 2000.

[5] M. R. Wilson and B. Henderson, Eds., Bacterial Disease Mechanisms, Cambridge University Press, Cambridge, UK, 2002.

[6] C. Pagnini, V. D. Corleto, S. B. Hoang, R. Saeed, F. Cominelli, and G. D. Fave, "Commensal bacteria and "oncologic surveillance": suggestions from an experimental model," Journal of clinical gastroenterology, vol. 42, supplement 3, part 2, pp. S193-S196, 2008.

[7] L. R. Johnson, Ed., Structure and Function of Gastrointestinal Mucus, Raven Press, New York, NY, USA, 1981.

[8] H. Florey, "Mucin and the protection of the body," Proceedings of the Royal Society of London B, vol. 143, no. 911, pp. 144-158, 1955.

[9] A. J. MacPherson, E. Slack, M. B. Geuking, and K. D. McCoy, "The mucosal firewalls against commensal intestinal microbes," Seminars in Immunopathology, vol. 31, no. 2, pp. 145-149, 2009.

[10] L. C. Tran and J. A. Di Palma, "Lack of lasting effectiveness of PEG 3350 laxative treatment of constipation," Journal of Clinical Gastroenterology, vol. 39, no. 7, pp. 600-602, 2005.

[11] V. Pampati and R. Fogel, "Treatment options for primary constipation," Current Treatment Options in Gastroenterology, vol. 7, no. 3, pp. 225-233, 2004.

[12] T. H. Chow, Y. Y. Lin, J. J. Hwang et al., "Therapeutic efficacy evaluation of 111In-labeled PEGylated liposomal vinorelbine in murine colon carcinoma with multimodalities of molecular imaging," Journal of Nuclear Medicine, vol. 50, no. 12, pp. 2073-2081, 2009.

[13] D. E. Corpet and G. Parnaud, "Polyethylene-glycol, a potent suppressor of azoxymethane-induced colonic aberrant crypt foci in rats," Carcinogenesis, vol. 20, no. 5, pp. 915-918, 1999.

[14] E. Dorval, J. M. Jankowski, J. P. Barbieux et al., "Polyethylene glycol and prevalence of colorectal adenomas: populationbased study of 1165 patients undergoing colonoscopy," Gastroenterologie Clinique et Biologique, vol. 30, no. 10, pp. 11961199, 2006.

[15] H. K. Roy, J. Gulizia, J. K. DiBaise et al., "Polyethylene glycol inhibits intestinal neoplasia and induces epithelial apoptosis in Apc(min) mice," Cancer Letters, vol. 215, no. 1, pp. 35-42, 2004.

[16] D. E. Corpet, G. Parnaud, M. Delverdier, G. Peiffer, and S. Tache, "Consistent and fast inhibition of colon carcinogenesis by polyethylene glycol in mice and rats given various carcinogens," Cancer Research, vol. 60, no. 12, pp. 3160-3164, 2000.

[17] S. Videla, A. Lugea, J. Vilaseca et al., "Polyethylene glycol enhances colonic barrier function and ameliorates experimental colitis in rats," International Journal of Colorectal Disease, vol. 22, no. 6, pp. 571-580, 2007.

[18] F. K. Bedu-Addo, P. Tang, Y. Xu, and L. Huang, "Effects of polyethyleneglycol chain length and phospholipid acyl chain composition on the interaction of polyethyleneglycolphospholipid conjugates with phospholipid: implications in liposomal drug delivery," Pharmaceutical Research, vol. 13, no. 5, pp. 710-717, 1996.

[19] K. Edwards, M. Johnsson, G. Karlsson, and M. Silvander, "Effect of polyethyleneglycol-phospholipids on aggregate structure in preparations of small unilamellar liposomes," Biophysical Journal, vol. 73, no. 1, pp. 258-266, 1997.

[20] M. Johnsson and K. Edwards, "Liposomes, disks, and spherical micelles: aggregate structure in mixtures of gel phase phosphatidylcholines and poly(ethylene glycol)-phospholipids," Biophysical Journal, vol. 85, no. 6, pp. 3839-3847, 2003.

[21] V. Valuckaite, O. Zaborina, J. Long et al., "Oral PEG 15-20 protects the intestine against radiation: role of lipid rafts," American Journal of Physiology, vol. 297, no. 6, pp. G1041G1052, 2009.

[22] L. Wu, O. Zaborina, A. Zaborin et al., "High-molecular-weight polyethylene glycol prevents lethal sepsis due to intestinal Pseudomonas aeruginosa," Gastroenterology, vol. 126, no. 2, pp. 488-498, 2004.

[23] H. Tlaskalova-Hogenova, R. Stepankova, T. Hudcovic et al., "Commensal bacteria (normal microflora), mucosal immunity and chronic inflammatory and autoimmune diseases," Immunology Letters, vol. 93, no. 2-3, pp. 97-108, 2004.

[24] J. B. Kaper, "Pathogenic Escherichia coli," International Journal of Medical Microbiology, vol. 295, no. 6-7, pp. 355-356, 2005.

[25] J. J. Mancuso, A. M. Larson, T. G. Wensel, and P. Saggau, "Multiphoton adaptation of a commercial low-cost confocal microscope for live tissue imaging," Journal of Biomedical Optics, vol. 14, no. 3, article 034048, 2009.

[26] A. Schutz, D. Schneidenbach, G. Aust, A. Tannapfel, M. Steinert, and C. Wittekind, "Differential expression and activity status of MMP-1, MMP-2 and MMP-9 in tumor and stromal cells of squamous cell carcinomas of the lung," Tumor Biology, vol. 23, no. 3, pp. 179-184, 2002.

[27] J. Goverman, L. A. Schick, and J. Newman, "The bundling of actin with polyethylene glycol 8000 in the presence and absence of gelsolin," Biophysical Journal, vol. 71, no. 3, pp. 1485-1492, 1996.

[28] D. Drenckhahn and T. D. Pollard, "Elongation of actin filaments is a diffusion-limited reaction at the barbed end and is accelerated by inert macromolecules," Journal of Biological Chemistry, vol. 261, no. 27, pp. 12754-12758, 1986.

[29] A. U. Jawhari, A. Buda, M. Jenkins et al., "Fascin, an actinbundling protein, modulates colonic epithelial cell invasiveness and differentiation in vitro," American Journal of Pathology, vol. 162, no. 1, pp. 69-80, 2003.

[30] E. Long, A. V. Capuco, D. L. Wood et al., "Escherichia coli induces apoptosis and proliferation of mammary cells," Cell Death and Differentiation, vol. 8, no. 8, pp. 808-816, 2001.

[31] B. Shankar, S. Krishnan, V. Malladi, A. Balakrishnan, and P. H. Williams, "Outer membrane proteins of wild-type and intimin-deficient enteropathogenic Escherichia coli induce Hep-2 cell death through intrinsic and extrinsic pathways of apoptosis," International Journal of Medical Microbiology, vol. 299, no. 2, pp. 121-132, 2009.

[32] V. K. Viswanathan, A. Weflen, A. Koutsouris, J. L. Roxas, and G. Hecht, "Enteropathogenic E. coli-induced barrier function alteration is not a consequence of host cell apoptosis," American Journal of Physiology, vol. 294, no. 5, pp. G1165G1170, 2008.

[33] P. M. S. Figueiredo, M. T. Furumura, L. Aidar-Ugrinovich et al., "Induction of apoptosis in Caco-2 and HT-29 human intestinal epithelial cells by enterohemolysin produced by classic enteropathogenic Escherichia coli," Letters in Applied Microbiology, vol. 45, no. 4, pp. 358-363, 2007. 


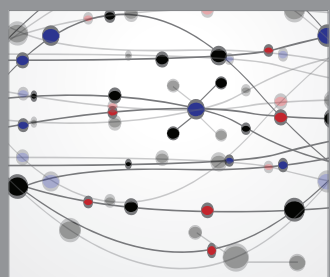

The Scientific World Journal
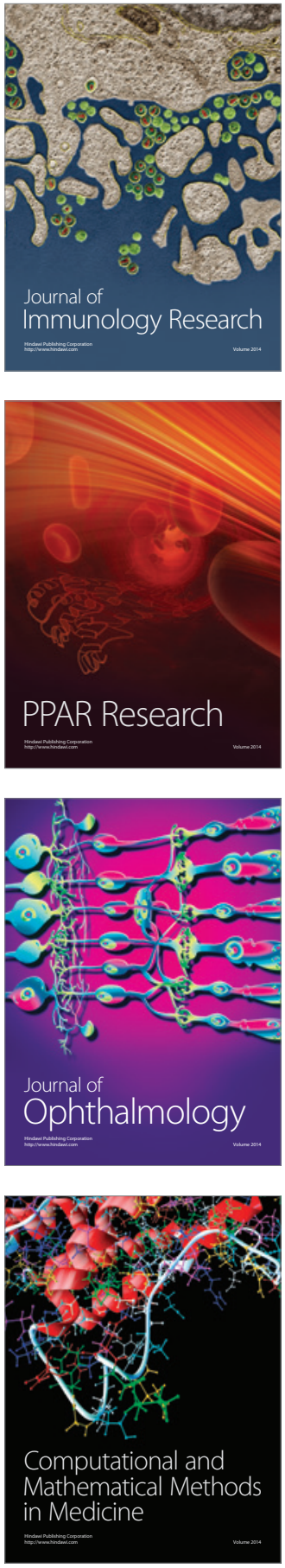

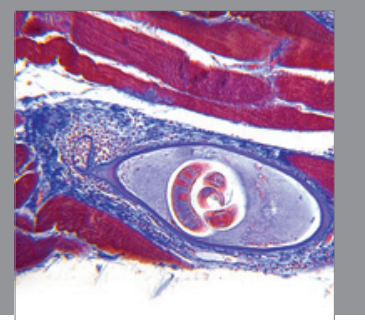

Gastroenterology

Research and Practice
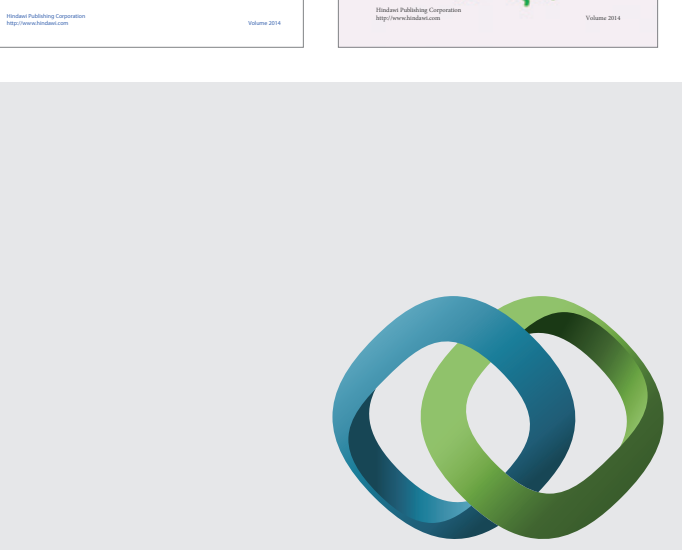

\section{Hindawi}

Submit your manuscripts at

http://www.hindawi.com
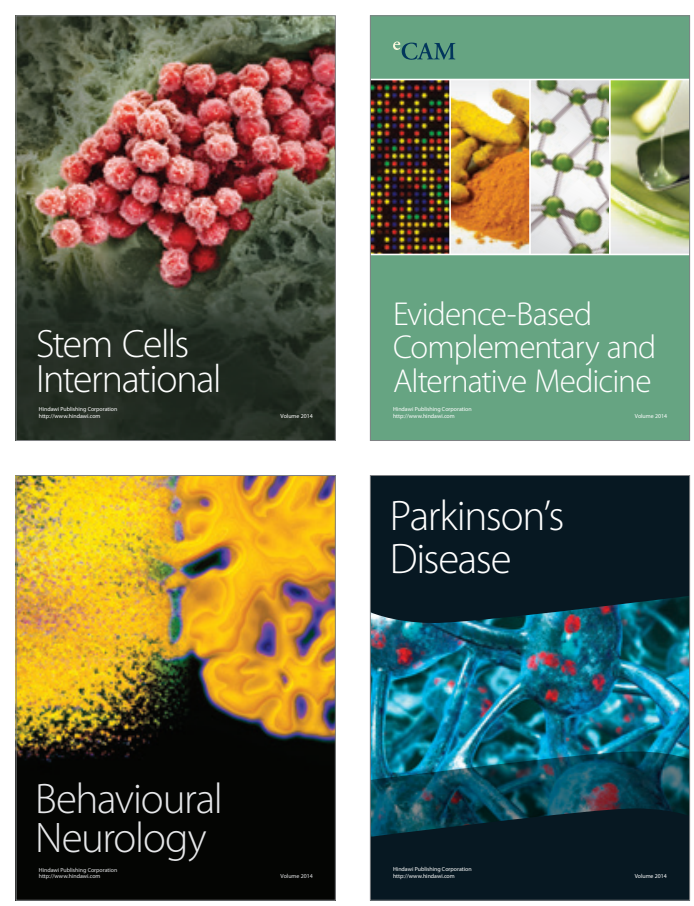

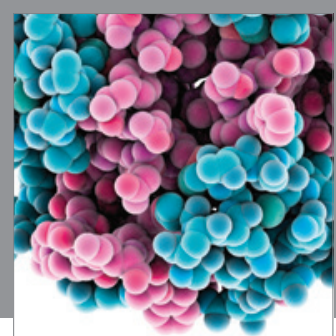

Journal of
Diabetes Research

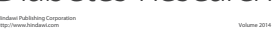

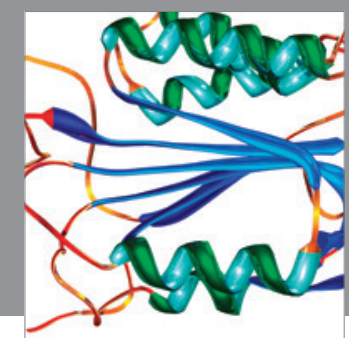

Disease Markers
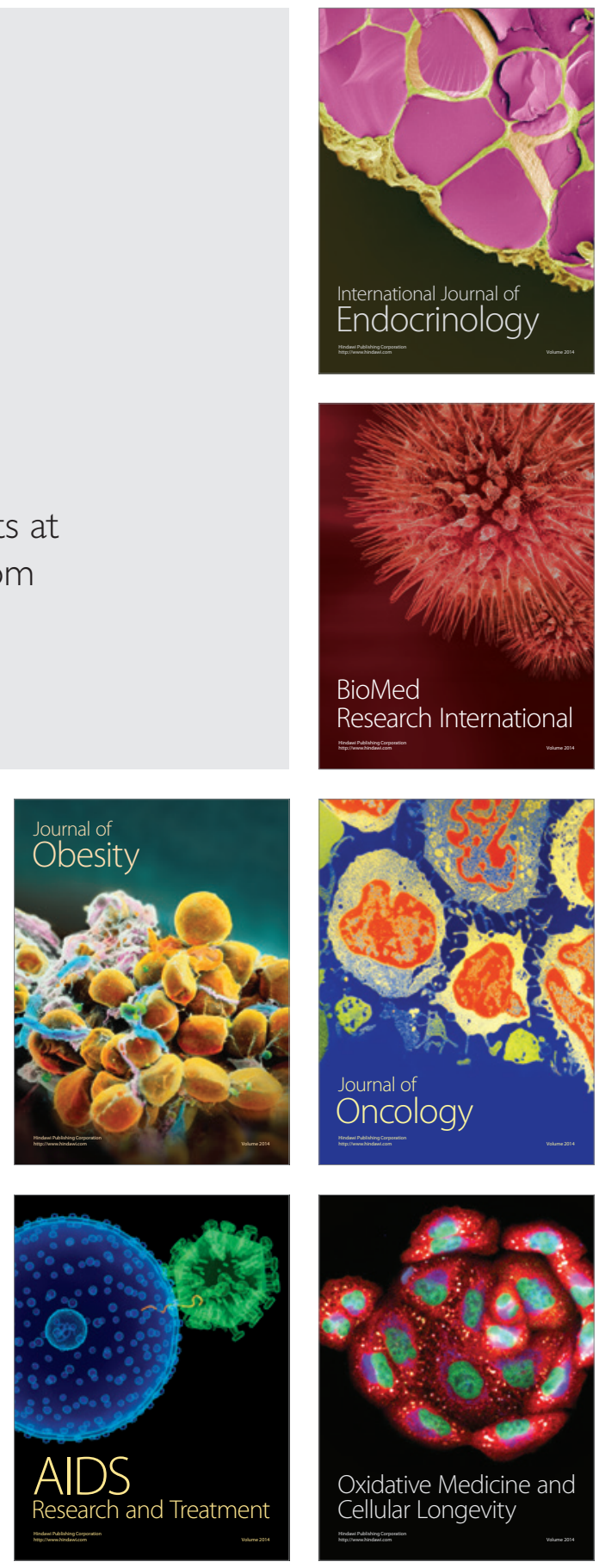